\title{
LETTER
}

doi:10.1017/S1041610212000439

\section{Caregiver burden of behavioral and psychological symptoms of dementia among Indian patients with Alzheimer's disease}

Behavioral and psychological symptoms of dementia (BPSD) are common in Alzheimer's disease (AD) and can potentially contribute to disease burden among caregivers. In this letter, an attempt is reported to establish if disease burden was higher among those with BPSD compared to those without it.

In 1996, the International Psychogeriatric Association Consensus Group defined BPSD as "symptoms of disturbed perception, thought content, mood or behaviour that frequently occur in patients with dementia." BPSD are common in AD (Coen et al., 1997). These are among the most important factors when considering whether a dementia patient should be institutionalized or not.

Western data suggest that BPSD occur in about 50-90\% of patients with dementia (Coen et al., 1997), while little is known about the prevalence of or associations with BPSD in developing countries (10/66 Dementia Research Group, 2004). Aggregate data of BPSD per se is not available in an Indian context. However, incidence of individual symptoms of BPSD is available e.g. between $10 \%$ and $73 \%$ of patients with $\mathrm{AD}$ experience delusions, with paranoid delusions being the most common. Similarly, hallucinations occur in 12$53 \%$ of patients with $\mathrm{AD}$. Visual hallucinations are most common followed by auditory hallucinations. Aggressive symptoms such as verbal outbursts, physical threats, or violence and agitation were observed in $75 \%$ of the patients (Shaji et al., 2009). There is a paucity of cross-cultural studies of BPSD.

Prevalence of $\mathrm{AD}$ in India appears to be low. Indians generally have joint family norms since ancient times as opposed to nuclear family norms in Western countries, which perhaps contribute to psychological as well as social support for the elderly. Culture can also influence BPSD and caregivers' response to such symptoms (Shaji et al., 2009). We therefore wanted to know if BPSD contribute to caregiver's burden in the Indian context, and, if so, to what extent.

The study was conducted in an outpatient department of a neurology unit of a tertiary care hospital from November 2006 to March 2007, in
New Delhi, India. Patients were recruited randomly and followed up prospectively. $\mathrm{AD}$ patients fulfilling National Institute of Neurological and Communicative Diseases and Stroke/Alzheimer's Disease and Related Disorders (NINCDS-ARDA) criteria (Ihl et al., 2011) were included in the study. Cooperative patients and their relatives willing to be interviewed for study were recruited.

Secondary causes of dementia were excluded by appropriate investigations, e.g. T3, T4, and TSH estimation, VDRL, FTS-Ab. The BEHAVE-AD scale (Schreinzer et al., 2005) was used to assess BPSD. The Pai and Kapur Disease Burden Scale (Pai and Kapur, 1981) was used to assess caregiver burden.

A total of 38 patients were studied $(\mathrm{M}: \mathrm{F}=33: 5$; age range $=55-85$ years $)$. Mini-Mental State Examination (MMSE) scores were calculated for each patient and they were divided into three dementia severity categories depending upon their score (mild- I $=18$; moderate- $\mathrm{II}=12$; severe- $\mathrm{III}=8$ ).

BPSD were found in $20(53 \%)$ out of 38 patients. Nine out of $20(45 \%)$ patients had psychotic symptoms (visual hallucinations $=4$; auditory hallucinations $=2$; delusions $=3$ ). Anxiety and phobias were seen in five patients. Anger outbursts were seen in three and two patients had violent behaviors. One patient had affective disturbance involving frequent crying and emotional outbursts. Fifteen out of the 20 were receiving treatment for BPSD while the rest did not receive any special treatment. Olanazpine and quetiapine were the two most commonly used drugs.

The Pai and Kapur Disease Burden Scale (Pai and Kapur, 1981) score was higher in those with BPSD compared to those without $(p<0.05)$. The MMSE score was lower in those with BPSD compared to those without ( $\mathrm{p}<0.05)$.

BPSD represent a growing burden to caregivers and healthcare institutions and contribute to the financial burden as well. There is increased frequency of depression and emotional problems in caregivers of AD patients (Schreinzer et al., 2005). The occurrence of BPSD is often the triggering event for recognition and referral of people with dementia to specialist services. These symptoms often present relatively late in the course of the illness. The development of BPSD is a major risk factor for caregiver burden (Coen et al., 1997) and institutionalization, and is more important in this regard than the enduring cognitive deficits of the disease process. All aspects of BPSD can be associated with caregiver burden, but paranoia, 
aggression, and sleep-wake cycle disturbance appear to be particularly important. The burden associated with BPSD is different for each symptom and does not always depend on frequency and severity of BPSD. It has been reported that some symptoms, such as agitation/aggression and irritability/lability, may affect the caregivers significantly, although their frequency and severity are low (Suh and Kim, 2004). Health policies to meet the unmet needs of elderly people are urgently needed, especially for AD patients at home without treatment (Matsumoto et al., 2007). The present study shows that the disease burden among those with BPSD is higher compared to those without it ( $p$ $<0.05$ ). Early recognition and prompt treatment of BPSD with antipsychotics can potentially decrease the caregiver burden and delay institutionalization of patients with $\mathrm{AD}$.

\section{Conflict of interest}

None.

\section{References}

Coen, R. F. et al. (1997). Distinguishing between patients with depression or very mild Alzheimer's disease using the Delayed-Word-Recall test. Dementia and Geriatric Cognitive Disorders, 8, 244-247.

Ihl, R. et al. (2011). WFSBP task force on treatment guidelines for Alzheimer's disease and other dementias.
World Federation of Societies of Biological Psychiatry (WFSBP) guidelines for the biological treatment of Alzheimer's disease and other dementias. World fournal of Biological Psychiatry, 12, 2-32.

Matsumoto, N. et al. (2007). Caregiver burden associated with behavioral and psychological symptoms of dementia in elderly people in the local community. Dementia and Geriatric Cognitive Disorders, 23, 219-224.

Pai, S. and Kapur, R. L. (1981). The burden on the family of a psychiatric patient: development of an interview schedule. British fournal of Psychiatry, 138, 332-335.

Schreinzer, D. et al. (2005). Components of behavioral pathology in dementia. International fournal of Geriatric Psychiatry, 20, 137-145.

Shaji, S., Bose, S. and Kuriakose, S. (2009). Behavioral and psychological symptoms of dementia: a study of symptomatology. Indian fournal of Psychiatry, 51, 38-41.

Suh, G. H. and Kim, S. K. (2004). Behavioral and Psychological Signs and Symptoms of Dementia (BPSD) in antipsychotic naive Alzheimer's disease patients. International Psychogeriatrics, 16, 337-350.

The 10/66 Dementia Research Group (2004). Dementia in developing countries. A preliminary consensus statement from the 10/66 Dementia Research Group. International fournal of Geriatric Psychiatry, 15, 14-20.

Vikas Dhikav AND Kuljeet Singh ANAND Department of Neurology, Postgraduate Institute of Medical Education and Research (PGIMER), Dr. Ram Manohar Lohia Hospital, GGS-IP University, New Delhi, India. Email: vikasdhikav@hotmail.com 